CASE STUDY

\title{
Therapaws: A Partnership Between Students, Staff, and Therapy Dogs on a University Campus
}

\author{
*Rebecca Johinke, Karen Walker, Freia Kirkaldy, Caitlyn Sinclair, Wing Lam (Kristi) Cheng, \\ Brian Tran, Emerald Williamson, Grace White, and Sara Sri Pillai, Faculty of Arts and Social \\ Sciences, Student Affairs and Engagement, University of Sydney, NSW, 2006, Australia
}

Contact: rebecca.johinke@sydney.edu.au

\begin{abstract}
Partnering with students in action research and asking them how and why they would like to work with staff and other students to improve campus culture and student wellbeing is the cornerstone of this case study. Investment in student mental health and wellbeing is increasingly recognised as a priority in higher education, with novel approaches such as dog therapy programs being introduced in universities around the world. This case study highlights a project where staff and students partner to codesign, co-implement, and co-investigate a mental health and wellbeing program that combines dog therapy with students-as-partners principles. The student-led dog therapy program (Therapaws) provides a practical, evidence-based example of how the principles of SaP can be employed to create an effective intervention into student mental health and wellbeing. This multi-authored case study is also an example of a collaborative writing process - a true partnership.
\end{abstract}

\section{KEYWORDS}

students as partners, dog therapy, student wellbeing, student mental health

This case study responds to the need for evidence-based, co-authored research on the impact of Students as Partners (SaP), which has been identified as a key priority in higher education (Healey, Flint, \& Harrington, 2014; Mercer-Mapstone et al., 2017). Existing SaP research and the principles of SaP demonstrate that SaP positively impacts students' confidence, engagement, and sense of shared responsibility and community (Healey, Flint, \& Harrington, 2014). The project centred on campus visits by specialist therapy dogs (and their handlers), peer-to-peer conversations about wellbeing, and campus support services. From start to finish, our team of staff (one academic and two professional) and six undergraduate student researchers have co-investigated the impact of dog therapy on our student volunteers 
and student participants. Additionally, this case study provides practical insights into studentstudent partnerships, which have been identified as another key priority for further research since student-student partnerships are the second most common partnership structure employed in practice, and yet are underexplored in research (Mercer-Mapstone et al., 2017). To that end, student-student partnerships are a key pillar of how the Therapaws program is designed to impact students by increasing knowledge of mental health, wellbeing and student support services.

ISSUE

Mental health and anxiety significantly affect university students in comparison to the general population, and are associated with decreased retention and academic performance. A World Health Organization survey of university students across 21 countries found an average of $20.3 \%$ of university students had long-term psychological illnesses (Auerbach et al., 2016). Only $16.4 \%$ of those university students with long-term psychological illnesses received mental health care, and a link to attrition was found (Auerbach et al., 2016). Within Australia, a crossinstitutional survey of psychological distress found that $83.9 \%$ of university students reported elevated distress levels, which was significantly higher than the $29 \%$ level of incidence found in the general Australian population (Stallman, 2010). Students with elevated distress levels were found to have more days away from their studies and a lower grade point average (Stallman, 2010). Similarly, stress, depression, and anxiety levels in Australian university students have been found to be higher than the Australian general population; for example, Lovell, Nash, Sharman, and Lane (2015) found mild or higher stress levels in $26.5 \%$ of university students, mild or higher depression levels in $21.8 \%$ of students, and mild or higher anxiety levels in $28.5 \%$ of students. Robotham and Julian (2006) identify a number of unique stressors that affect university students compared to the general population: examination stress, financial pressures, transition to university, and study-related stress. Stigma and lack of knowledge about student support services have been identified as significant barriers to accessing the psychological support that universities provide (Holland, 2016; Yorgason, Linville, \& Zitzman, 2008). There is a need for additional student wellbeing support that breaches these gaps by targeting these unique stressors and barriers. One of the key findings in Veness' (2016) Churchill Fellowship report, The Wicked Problem of University Student Mental Health, is that students must be part of the solution, with student representatives as key partners in the development of policies and intervention strategies.

\section{STRATEGY}

Dog therapy provides a relatively stigma-free, low-cost, engaging mental health intervention, which is supported by an emerging body of research that suggests efficacy in reducing stress (Daltry \& Mehr, 2015; S. McDonald, E. McDonald, \& Roberts, 2017; Muckle \& Lasikiewicz, 2017; Ward-Griffin et al., 2018). Therapaws pairs dog therapy with the principles of SaP to amplify the impact of the program on student wellbeing by targeting these unique student stressors and barriers. Student-staff partnerships are critical, with a particular value placed on the ability of students to know what kind of program would best engage their peers, 
what specific stressors students are facing, what information students do not have, and how to communicate that information in a student-friendly manner. Professional and academic staff members provide the expertise, time commitment, and resources needed to organise largescale, complex programs and ensure student partners receive training and mentoring to build upon their existing skills. Student-student partnerships are also critical, with student volunteers functioning as visible, peer ambassadors for positive mental health through conversation with student participants as part of the dog therapy program.

In designing the project, the latest scholarship on students-as-partners is primarily based on Healey, Flint, and Harrington's (2014) model and the work coming out of McMaster University. Given that this project is extracurricular, we employed a simplified students-aspartners model, as conceptualised by Healey, Bovill, and Jenkins (2015) and illustrated below in Figure 1, rather than one of the more complex partnership models that currently inform the curriculum. The group felt these models did not adequately cater to an extracurricular project that is aligned with the attainment of overall graduate qualities related to leadership and influence rather than classroom teaching. In terms of Scholarship of Teaching and Learning (SoTL) and SaP principles, there is the potential, we believe, to re-examine the current suite of SaP conceptual models to better incorporate small-scale extracurriculum projects that are so often part of a SaP program (Mercer-Mapstone et al., 2017).

\section{Figure 1: Students as partners}

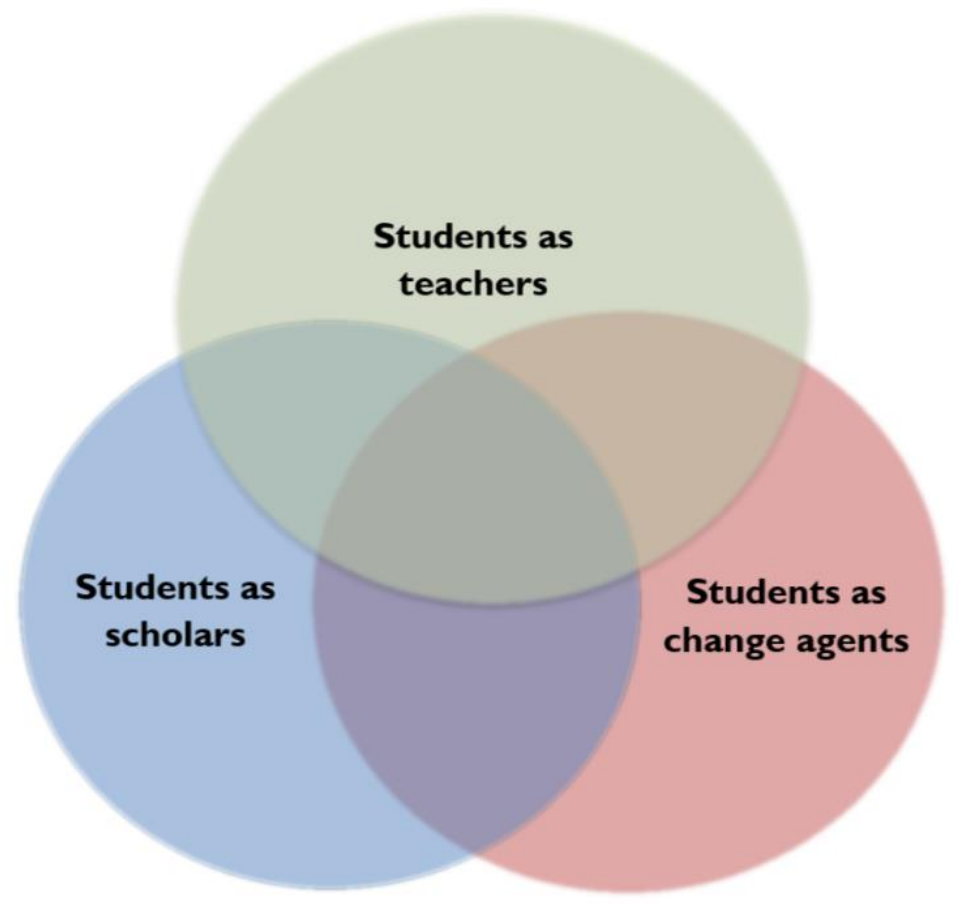

Source: Healey, Bovill, \& Jenkins (2015, p. 142).

Simply stated, we were committed to the principles of students as teachers (student to student primarily but also student to staff), students as scholars (based on the SaP model, 
engaging around student wellbeing and the possible benefits of dog therapies), and students as change agents (affecting campus culture). Given that the academic lead's disciplinary background is literary and rhetorical studies, the work of Ede and Lunsford (1990) on the collaborative writing process is also embedded in the project's approach to writing and research as an iterative process that involves trust, reciprocity, honesty, courage, and responsibility (all principles and values of partnerships espoused by Healey, Flint, and Harrington, 2014).

\section{INSTITUTIONAL CONTEXT}

Within this framework, students and staff co-designed four key program aims, which guided us throughout our partnership. Therapaws aimed to:

1. contribute to a healthy learning environment for students by reducing stress through dog therapy;

2. facilitate peer-to-peer messages regarding mental health and wellbeing and connect students with information such as counselling and psychological services, disability services, peer mentoring, student representation, and academic advice services;

3. increase student engagement with the Faculty of Arts and Social Sciences and contribute to creating a sense of identity and community; and

4. create opportunities for students to act as change agents in the university and gain graduate qualities relevant to their personal, professional, and academic futures.

\section{PROGRAM CO-DESIGN, CO-IMPLEMENTATION, AND CO-INVESTIGATION}

Our partnership began with Healthy Sydney University, an institution-wide strategy at the University of Sydney that enabled the co-creation of Therapaws by providing seed funding for new staff-student health and wellbeing initiatives. In mid-2016, staff sent a call-out to our students, asking for expressions of interest to work with us on a SaP project. Interested students attended a group planning meeting with staff to brainstorm ideas for initiatives. Dog therapy was suggested as a potential project; the idea excited the group and was backed up by research as an effective means of reducing stress. An undergraduate student signed on as the student lead to apply for the grant, and 13 additional students signed up for general volunteering. An academic staff member agreed to sponsor the project and act as the academic lead.

Staff and students worked together to co-design the program, write the grant application, and trial the idea with a one-off Therapaws session in late 2016. The trial was successful and a AUD\$2,500 Healthy Sydney University grant was awarded to scale up the program in 2017. Three more students joined the student lead on the core student leadership team who would take on a high level of responsibility for the program co-design, serve as peer leaders among the wider student volunteer team, and work closely with staff to conduct research on the impact of the program. Although we were enormously grateful to receive the funding, the grant was relatively modest and so all funds were used to pay the dog owners for their time, with students volunteering their time. Ethics approval was granted in early 2017, and five Therapaws sessions were held throughout the year. The program primarily worked through three key 
activities: planning meetings, undertaking Therapaws sessions, and completing the research project. We attribute the success of these three key activities and the wider program to the principles of SaP (with its emphasis on shared responsibility, ownership, and decision making), the use of dialogue for building relationships and sharing power, and the use of partnership as a process where all participants stand to gain from learning and working together (Healey, Flint, \& Harrington, 2014; Matthews, 2017).

Regular, small group planning meetings involved the student volunteer team, student leadership team, and staff. In these meetings, we discussed ideas and made decisions about how the program and individual therapy sessions would be designed, and marketing and advertising. In the trial stage leading up to our first session, we met weekly to make larger-scale decisions about what the program should look like. In the implementation stage, we met once in the fortnight leading up to each session to reflect on the previous session, discuss what we wanted to improve next time, identify a theme for each session based on student needs at that time in semester, plan the information on mental health and wellbeing that would be included in the conversations and information handout, plan marketing and communications, and allocate specific responsibilities to prepare at home for each session and for the actual sessions.

Therapaws sessions lasted 90 minutes, with two hours allocated beforehand for volunteer briefing and set-up, and one hour afterwards for pack-down and reflection. Student volunteers first met for a briefing conducted by staff and the student leadership team, which involved reviewing the run sheet for the day, reviewing the health and wellbeing handout, and undergoing training on talking to students about health and wellbeing. Students and staff then began setting up each session in an outdoor location with high visibility and foot traffic, as well as facilitating the arrival of the therapy dogs and handlers from our not-for-profit partner, Delta Society Australia, a leading national provider of dog therapy. Delta Society Australia provided trained therapy dogs and handlers and technical expertise on animal welfare and stressreduction through dog therapy.

Sessions were organised around two key activities. First, five handlers and therapy dogs spread out over a large lawn, with five to 10 students in a group sitting around the therapy dogs and patting them. Students were free to drop in and out or stay the whole session. Second, members of our student volunteer team engaged student participants in conversations about mental health, wellbeing and student support, and gave them copies of the health and wellbeing handout to take home and read. The majority of student volunteers were engaged in these peer-to-peer conversations, with approximately five more producing content for social media, managing our health and wellbeing wall (an interactive canvas where students were encouraged to write positive messages to each other), managing fundraising bake sales, taking photos, and producing videos. Our student leadership team provided peer leadership roles, primarily supporting other students to engage in peer-to-peer conversations, produce engaging social media content and videos, troubleshoot problems, and even engage in peer-to-peer conversations themselves. Academic and professional staff from the faculty and wider university also attended to engage students in conversation, support the student volunteers, and organise logistics. Following the sessions, students and staff returned to the office where

Johinke, R., Walker, K., Kirkaldy, F., Sinclair, C., Cheng, W.L., Tran, B., Williamson, E., White, G., \& Sri 100 Pillai, S. (2018). Therapaws: A partnership between students, staff, and therapy dogs on a university campus. International Journal for Students as Partners, 2(2). 
students completed an optional post-session volunteer survey and students and staff reflected on the successes and challenges of each session over afternoon tea.

\section{PROGRAM IMPACT}

\section{Research project (students as scholars, researchers, and change agents)}

Essentially, we were conducting two streams of research. The first, and the one that was of most interest to students, was to ascertain whether Therapaws had had a positive impact on student wellbeing. Our first three program aims related to the impact of Therapaws on the mental health of the student participants who attended each session. These aims were primarily measured through quantitative data collected in our online student health and wellbeing survey, distributed to students who attended via social media. In addition to this survey, we measured the impact on student participants through attendance data, social media data, and analysis of the written content of our health and wellbeing wall from each session. Quantitative and qualitative evidence supports the claim that sessions had a positive impact. For example, $97 \%$ of student participants reported feeling less stressed after attending a Therapaws session and students left positive messages for each other (for example, "don't be afraid to seek help! You deserve to feel better and love yourself").

The second research piece-potentially of more interest to staff and definitely more important for readers of this case study-was whether the project had been successful in terms of SaP. To that end, in addition to their roles in the planning meetings and Therapaws sessions, the student leadership team partnered with staff to co-investigate the impact of the Therapaws program on the student volunteers and the students who participated in sessions. This related to our fourth key program aim, which was to give students the opportunity to act as change agents in the university and gain graduate skills and experience. Central to student learning at the University of Sydney is that a Faculty of Arts and Social Sciences graduate should be effective in exercising professional and social responsibility and making a positive contribution to society, in addition to having applied disciplinary expertise, confidence, personal resilience, and broad critical thinking, problem solving, and communication skills ("Graduate Qualities," 2018). We analysed the impact of Therapaws on our student partners with reference to this framework through two student volunteer surveys, which students completed before $(n=33)$ and after volunteering at each session $(n=63)$. This group of student leaders-already identified as empowered and capable-consistently self-perceived their skills and abilities as high, with no statistically significant quantitative difference observed between the before and after responses. However, we believe our qualitative data provide evidence on the impact of $\mathrm{SaP}$ on the volunteers - by creating opportunities for students to act as change agents, the students can become engaged, socially responsible citizens who can make a positive impact on the mental health and wellbeing of their peers. The students also benefited from the program through skill development. The skills the students most commonly identified were communication skills (84\%), confidence, teamwork, and interpersonal skills (71\%), and critical thinking, organisational, and planning skills (28\%). One student identified having "better 
communication skills, organisational skills, experience in seeing how events are managed and the structure required to [make] them a success."

\section{CHALLENGES}

Happily, we believe we managed to avoid many of the challenges discussed in the SaP literature (Mercer-Mapstone et al., 2017). However, despite the success of the project as both a partnership program and a mental health and wellbeing initiative, Therapaws is what Healey and Jenkins (2009) would classify as an elite model-a one-off, small-scale project made possible by a seed funding grant and hence not sustainable (Mercer-Mapstone et al., 2017). As already noted, the dog handlers were compensated for their time but students were not, and non-payment of students has been raised as an issue for students who cannot afford to volunteer their time and labour (Mercer-Mapstone et al., 2017). Moreover, it proved to be an expensive program to run in terms of the staff time required to work collaboratively with the undergraduate students in organising and managing the dog therapy sessions as well as conducting research and working on the writing process. These latter tasks included ethics approvals, survey design, data interpretation, crafting conference papers, and preparing this case study. As has been noted in the SaP literature, time constraints and the pressure to publish also factor into such partnerships (Mercer-Mapstone et al., 2017).

The benefits are clear but they do come at a price. Dedicated grant money or sponsorship would be required to continue with this SaP project or it would need to be redesigned as a project-based unit of study and embedded in the curriculum. This embedded approach is clearly favoured by Healey, Flint, and Harrington (2014) and other SaP scholars, as the benefits can be shared by more students (Mercer-Mapstone et al., 2017). However, as student partnerships are being increasingly mainstreamed as part of our institution's curriculum transformation process, this case study signals the end of the project for the time being. We are hopeful that other dog therapy or student-led wellness initiatives will flourish at our institution in the near future. Alternatively, a low- or no-cost alternative worth investigating at other institutions might include bring your dog to campus days (much like many workplaces now facilitate) or other forms of SaP collaboration that do not involve dog therapy (such as student involvement in curriculum reform).

\section{CONCLUSION}

Our case study provides a practical, evidence-based example of how the principles of SaP can be applied to create an engaging and effective intervention into student mental health and wellbeing. It offers an insight into the benefits of student-student partnerships, an area that has been identified as under-researched in the SaP literature. We believe there are four key significant results that suggest Therapaws as a model for future SaP programs targeting student wellbeing. First, the high levels of student engagement are a testament to the studentstaff and student-student partnerships that underpin Therapaws. Second, student-student partnerships were successful in increasing student knowledge about student support services. Third, student-student partnerships through social media were a particularly valuable way to engage students in conversations about mental health. Finally, Therapaws successfully created 
opportunities for students to act as change agents, with $91 \%$ of student volunteers believing they had made a positive impact on student mental health and wellbeing. Based on these results, we recommend that future dog therapy programs or similar mental health and wellbeing programs incorporate the principles of SaP as a model of good practice. SaP amplifies the impact of university-based dog therapy programs beyond the stress reduction effects of dog therapy itself. Moreover, as with any SaP initiative, it is the process of engagement, not the product or outcome, that is just as important in this case study.

This research has been approved by the University of Sydney Research Ethics Committee.

\section{ACKNOWLEDGEMENTS}

The authors acknowledge the generosity of Healthy Sydney University for the original seed funding, the wonderful dog handlers from the Delta Society Australia, and the support of our Dean, Professor Annamarie Jagose.

\section{NOTE ON CONTRIBUTORS}

Rebecca Johinke is the Associate Dean, Student Affairs for the Faculty of Arts and Social Sciences at the University of Sydney and an associate professor in the Department of English.

Karen Walker is the Head, Student Affairs and Engagement for the Faculty of Arts and Social Sciences, University of Sydney.

Freia Kirkaldy, is the Student Affairs and Engagement Coordinator for the Faculty of Arts and Social Sciences and managed Therapaws.

Caitlyn Sinclair is a BA (Media and Communications) student at the University of Sydney who now works with the Student Affairs and Engagement team in the Faculty of Arts and Social Sciences. She was part of the Therapaws Student Leadership Team.

Wing Lam (Kristi) Cheng is a BA (Media and Communications) student, majoring in Chinese and French Studies. She is the Healthy Sydney University scholar and part of the Therapaws student leadership team.

Brian Tran is a recent BA and BS graduate who majored in digital cultures and nutrition and metabolism. He was part of the Therapaws student leadership team.

Emerald Williamson is a Bachelor of International and Global Studies student, majoring in international business, and was part of the Therapaws student leadership team.

Grace White is a BA and BS student, majoring in Indigenous Australian studies, and was part of the Therapaws student leadership team. 
Sara Sri Pillai is a Bachelor of Liberal Arts and Science student, majoring in English and computer science, and was part of the Therapaws student leadership team.

\section{REFERENCES}

Auerbach, R. P., Alonso, J., Axinn, W. G., Cuijpers, P., Ebert, D. D., Green, J. G., \& Bruffaerts, R. (2016). Mental disorders among college students in the world health organization world mental health surveys. Psychological Medicine, 46(14), 2955-2970. http://dx.doi.org/10.1017/S0033291716001665

Daltry, R. M., \& Mehr, K. E. (2015). Therapy dogs on campus: Recommendations for Counselling Centre Outreach. Journal of College Student Psychotherapy, 29(1), 72-78. http://dx.doi.org/10.1080/87568225.2015.976100

Ede, L., \& Lunsford, A. (1990). Singular texts/plural authors: Perspectives on collaborative writing. Carbondale, IL: Southern Illinois University Press.

Faculty of Arts and Social Sciences, University of Sydney. (2017). Graduate qualities. Retrieved from http://sydney.edu.au/arts/teaching learning/academic support/graduate qualities.sht $\underline{\mathrm{ml}}$

Healey, M., Bovill, C., \& Jenkins, A. (2015). Students as partners in learning. In Lea, J. (Ed.) Enhancing Learning and Teaching in Higher Education: Engaging with the Dimensions of Practice. Milton Keynes: Open University Press.

Healey, M., Flint, A., \& Harrington, K. (2014). Engagement through partnership: students as partners in learning and teaching in higher education. York: Higher Education Academy. Retrieved from https://www.heacademy.ac.uk/system/files/resources/engagement through partnersh ip.pdf

Healey, M., \& Jenkins, A. (2009). Developing undergraduate research and inquiry.York: Higher Education Academy. Retrieved from www.heaacademy.ac.uk/assets/York/documents/resources/publications/DevelopingUn dergraduate Final.pdf

Holland, D. (2016). College student stress and mental health: Examination of stigmatic views on mental health counselling. Michigan Sociological Review, 30, 16-43.

Lovell, G. P., Nash, K., Sharman, R., \& Lane, R. B. (2015). A cross-sectional investigation of depressive, anxiety, and stress symptoms and health-behaviour participation in Australian university students. Nursing and Health Sciences, 17, 134-142. http://dx.doi.org/10.1111/nhs.12147

Matthews, K. E. (2017). Five propositions for genuine students as partners practice. International Journal for Students as Partners, 1(2), 1-9. http://dx.doi.org/10.15173/ijsap.v1i2.3315 
McDonald, S., McDonald, E., \& Roberts, A. (2017). Effects of novel dog exposure on college students' stress prior to examination. North American Journal of Psychology, 19(2), 477484.

Mercer-Mapstone, L., Dvorakova, S. L., Matthews, K. E., Abbot, S., Cheng, B., Felten, P., Knorr, K., Marquis, E., Shammas, R., \& Swaim, K. (2017). A systematic literature review of students as partners in higher education. International Journal for Students as Partners, 1(1), 1-23. http://dx.doi.org/10.15173/ijsap.v1i1.3119

Muckle, J., \& Lasikiewicz, N. (2017). An exploration of the benefits of animal-assisted activities in undergraduate students in Singapore. Asian Journal of Social Psychology, 20, 75-84. http://dx.doi.org/10.1111/ajsp.12166

Robotham, D., \& Julian C. (2006). Stress and the higher education student: a critical review of the literature. Journal of Further and Higher Education, 30(2), 107-117. http://dx.doi.org/10.1080/03098770600617513

Stallman, H. M. (2010). Psychological distress in university students: A comparison with general population data, Australian Psychologist, 40(4), 249-257. http://dx.doi.org/10.1080/00050067.2010.482109

Veness, B. G. (2016). The wicked problem of university student mental health. Report to the Winston Churchill Memorial Trust. Sydney, Australia.

Ward-Griffin, E., Klaiber, P., Collins, H. K., Owens, R. L., Coren, S., \& Chen., F. S. (2018). Petting away pre-exam stress: The effect of therapy dog sessions on student well-being. Stress and Health, 1-6. http://dx.doi.org/10.1002/smi.2804

Yorgason, J. B., Linville, D., \& Zitzman, B. (2008). Mental health among college students: Do those who need services know about and use them? Journal of American College Health, 57(2), 173-81. 